\title{
Interface manipulated two-phase nanostructure in a triblock terpolymer with a short middle segment
}

\author{
Rina Maeda ${ }^{1,7}$, Takeshi Higuchi ${ }^{2,8}$, Kenta Okuhara ${ }^{1}$, Ryohei Kikuchi ${ }^{3}$, Atsushi Takahara ${ }^{2,4}$, \\ Christopher K Ober ${ }^{5}$, Hiroshi Jinnai ${ }^{2,8}$ and Teruaki Hayakawa ${ }^{1,6}$
}

Interface manipulation to pursue unusual nanostructures was performed using a strategically designed triblock terpolymer with a short middle segment, poly(styrene- $b$-methyl methacrylate- $b$-2,2,2-trifluoroethyl methacrylate). A short middle segment of poly (methyl methacrylate) (PMMA) that does not form any distinct domains was found to play an important role in manipulating the interface between the polystyrene (PS) and poly(2,2,2-trifluoroethyl methacrylate) (PTFEMA) domains and forming unconventional partially continuous oblate cylinders with an oblique lattice, as confirmed by small angle X-ray scattering (SAXS) and dual-axis transmission electron microtomography (TEMT).

Polymer Journal (2016) 48, 533-538; doi:10.1038/pj.2016.25; published online 24 February 2016

\section{INTRODUCTION}

It is important to form a wide variety of morphologies based on microphase separation in block copolymers. A simple yet flexible polymer design for the formation of a wide variety of shapes and periodicities of microphase-separated structures could be utilized in numerous nanostructured materials, such as energy materials, optoelectronic materials, and block copolymer lithography resists. ${ }^{1-4}$

There have been several studies for creating unique morphologies of block copolymers. For example, triblock terpolymers have been attracting much attention. ${ }^{5-8}$ In addition to temperature variation, the addition of low molecular weight solvents to block copolymers, homopolymers, or other block copolymers have also been shown to change morphologies. ${ }^{9}$ Furthermore, there have been reports on star-shaped, ${ }^{10}$ branched, ${ }^{11,12}$ and comb-shaped block polymers, ${ }^{13}$ and temperature and light-induced self-assembly changeable tetra-arm block copolymer, ${ }^{14}$ indicating that a large field has opened up new ways for manipulating morphologies.

Block copolymers modified at the junction point is another interesting system to obtain a wider variety of morphologies. For example, tapered block copolymers consisting of $\mathrm{AB}$ diblock copolymers with a random $\mathrm{A} / \mathrm{B}$ transition region between $\mathrm{A}$ and $\mathrm{B}$ segments potentially form intriguing morphologies and provide characteristic properties not attainable in conventional diblock copolymers. ${ }^{15-22}$ Hashimoto and coworkers investigated the morphologies of poly (styrene- $b$-styrene/isoprene- $b$-isoprene) with a styrene/isoprene tapered part where a tapered middle block segment incorporated. On the basis of their SAXS results and two-dimensional (2D) transmission electron microscopy (TEM) images, the morphology of the tapered block copolymer was suggestive of an unusual morphology, although not fully characterized, whereas the corresponding non-tapered block copolymer with the same molecular weight and composition showed simple lamellar structure. ${ }^{17}$ Epps and coworkers also found that the tapered interface led to lower order-to-disorder transition temperature of the gyroid structure relative to the corresponding non-tapered block copolymers. ${ }^{20-22}$ These studies have led to the realization that interfacial modification due to the introduction of a tapered part constitutes an additional parameter of the block copolymer nanostructures through interfacial thermodynamics.

There have been limited chemical structures of the block copolymers that are capable of manipulating the interfaces. Hashimoto and coworkers reported poly(isoprene- $b$-styrene) with a wellcontrolled tapered interfacial region using an anionic polymerization technique. ${ }^{16,17}$ Epps and coworkers also reported block copolymers with a tapered interfacial region, including an inverse tapered region, using a controlled monomer injection. ${ }^{20,21}$

The present work focuses on a new approach to create unique morphologies based on two-phase microphase-separated

\footnotetext{
${ }^{1}$ Department of Organic and Polymeric Materials, Tokyo Institute of Technology, Tokyo, Japan; ${ }^{2}$ Institute for Materials Chemistry and Engineering (IMCE), Japan Science and Technology Agency (JST) and ERATO, Takahara Soft Interfaces Project, Kyushu University, Fukuoka, Japan; ${ }^{3}$ Center for Advanced Materials Analysis (CAMA), Tokyo Institute of Technology, Tokyo, Japan; ${ }^{4}$ International Institute for Carbon-Neutral Energy Research (WPI-I2CNER), Kyushu University, Fukuoka, Japan; ${ }^{5}$ Department of Materials Science and Engineering, Cornell University, Ithaca, New York, USA and ${ }^{6}$ Precursory Research for Embryonic Science and Technology, Japan Science and Technology Agency, JST-PRESTO (Molecular Technology and Creation of New Functions), Saitama, Japan

${ }^{7}$ Current address: Department of Advanced Materials Science, Graduate School of Frontier Sciences, The University of Tokyo, 5-1-5 Kashiwanoha, Kashiwa-shi, Chiba 277-8561, Japan.

${ }^{8}$ Current address: Institute of Multidisciplinary Research for Advanced Materials, Tohoku University, 2-1-1 Katahira, Aoba-ku, Sendai 980-8577, Japan.

Correspondence: Professor H Jinnai, Institute of Multidisciplinary Research for Advanced Materials, Tohoku University, 2-1-1 Katahira, Aoba-ku, Sendai 980-8577, Japan. E-mail: hjinnai@tagen.tohoku.ac.jp

or Professor T Hayakawa, Department of Organic and Polymeric Materials, Tokyo Institute of Technology, 2-12-1-S8-36, Ookayama, Meguro-ku, Tokyo 152-8552, Japan. E-mail: hayakawa.t.ac@m.titech.ac.jp

Received 14 December 2015; revised 8 January 2016; accepted 16 January 2016; published online 24 February 2016
} 
nanostructures using a triblock terpolymer poly(styrene- $b$-methyl methacrylate- $b$-2,2,2-trifluoroethyl methacrylate). PMMA and PTFEMA provide selective degradability under either deep-UV (DUV) or e-beam radiation, leaving crosslinked PS domains behind, as reported by our previous study. ${ }^{23}$ The target material consists of two longer block segments, PS and PTFEMA, and one shorter block segment, PMMA, between them. The material can be synthesized in a conventional living anionic polymerization. The PMMA segment is short enough so that it does not form any distinct domain but plays an important role in manipulating the interface between the two phases composed of two other longer block segments, such as the tapered segment. We expected this new design of a triblock terpolymer to offer some advantages in its versatility of the synthesis and nanostructure design. Many conventional triblock terpolymers prepared by general living polymerization can be applied to this approach and are expected to yield unique nanostructures that are not obtainable in conventional diblock copolymers.

We herein describe the synthesis and bulk morphology of a novel triblock terpolymer poly(styrene- $b$-methyl methacrylate- $b-2,2$, 2-trifluoroethyl methacrylate) with the composition $\mathrm{PS}_{54} \mathrm{PMMA}_{15} \mathrm{PT}$ $\mathrm{FEMA}_{31}{ }^{38.1}$, where the subscripts denote the volume fraction of the corresponding block, and the superscript denotes the overall molecular weight in $\mathrm{kg} / \mathrm{mol}$. For the composition reported here, an oblique-packed cylindrical structure was found via a combination of SAXS, TEM and TEMT. ${ }^{24}$ The cylindrical domains consist of PTFEMA located in other components, which was effectively observed by dual-axis TEMT. A porous film was obtained by degradation of the PMMA and PTFEMA segments.

\section{EXPERIMENTAL PROCEDURE}

\section{Materials}

Chemicals were obtained from TCI, Sigma-Aldrich, Kanto Chemical, Wako Chemical or Alfa Aesar and were used without further purification unless otherwise noted.

For anionic polymerization, dehydrated stabilizer-free tetrahydrofuran (THF) purchased from Kanto Chemical was titrated with sec-BuLi at $-78^{\circ} \mathrm{C}$ and was distilled at room temperature just before polymerization. Monomers of styrene, methyl methacrylate (MMA) and 2,2,2-trifluoroethyl methacrylate (TFEMA) were successively stirred and distilled with calcium hydride. These monomers were distilled again under vacuum over a small amount of di- $n$-butylmagnesium (styrene) and tri- $n$-octylaluminum (MMA and TFEMA)

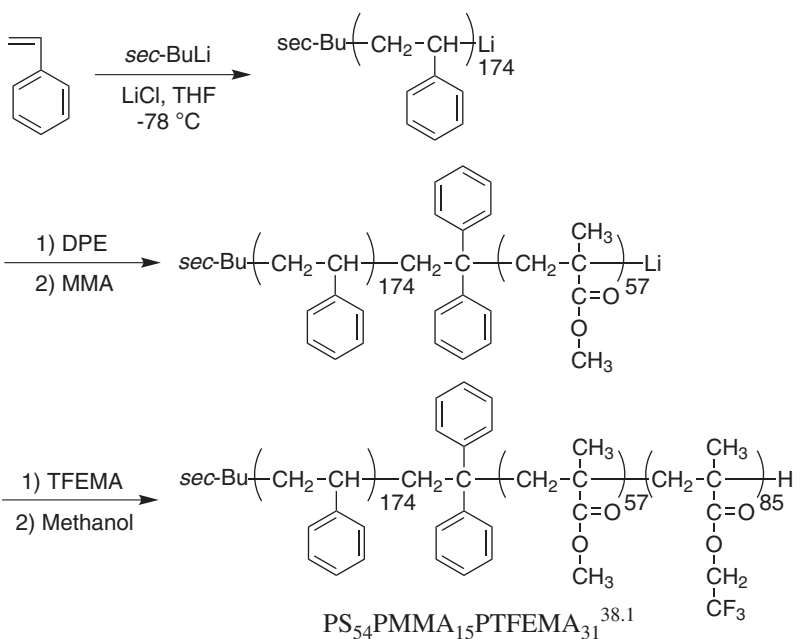

Figure 1 Synthetic route of the triblock terpolymer, $\mathrm{PS}_{54} \mathrm{PMMA}_{15}$ PTFEMA $_{31} 38.1$. just prior to use. 1,1-Diphenylethylene (DPE) was distilled over 1,1-diphenyl-3methylpentyllithium and was stored at $0{ }^{\circ} \mathrm{C}$.

\section{Polymer synthesis}

The polymer samples used in this study were prepared via sequential anionic polymerization of styrene, methyl methacrylate and 2,2,2-trifluoroethyl methacrylate, as illustrated in Figure 1. The typical synthetic procedure of the block copolymer is as follows. A $40 \mathrm{ml}$ volume of freshly distilled THF was transferred to a glass reactor containing $10 \mathrm{mg}$ of dry $\mathrm{LiCl}$, and the glass reactor was cooled to $-78^{\circ} \mathrm{C}$. Then, $76 \mu \mathrm{l}$ of sec-BuLi $(0.13 \mathrm{~m} \mathrm{sec}$-butyllithium in cyclohexane) was injected, and $2 \mathrm{ml}$ of styrene was added. After $10 \mathrm{~min}$ of polymerization, an aliquot of PS was isolated for analysis after termination with degassed methanol, and styryl anions were capped with $50 \mu \mathrm{l}$ of DPE, which resulted in a deep red color of the reaction mixture. Half an hour later, the polymerization was resumed by injection of $0.28 \mathrm{ml}$ of MMA with vigorous stirring, which led to immediate disappearance of the red color. After half an hour, $1 \mathrm{ml}$ of PTFEMA was added with vigorous stirring. After $30 \mathrm{~min}$, the reaction was terminated with $1 \mathrm{ml}$ of degassed methanol. After some of the THF was evaporated, the polymer was precipitated in $500 \mathrm{ml}$ of methanol and was dried under vacuum at $60^{\circ} \mathrm{C}$ for $8 \mathrm{~h}$.

\section{Polymer analysis}

The molecular weight characteristics of the PS precursor were determined by gel permeation chromatography (GPC) using THF as a solvent on a JASCO HBPX 800Pu and two polystyrene divinylbenzene columns (Shodex GPC 803L and $804 \mathrm{~L}$ ) with a refractive index detector based on a calibration curve using PS standards (purchased from Shodex). The chemical structure was confirmed by ${ }^{1} \mathrm{H}$ NMR $(400 \mathrm{mHz})$ and ${ }^{13} \mathrm{C}$ NMR $(100 \mathrm{mHz})$ spectra on a JEOL ECS400 spectrometer. The volume fraction of each block was calculated from elemental analysis on a J-Science JM10 (for carbon) and Yanaco HSU-20+ICS-1100 (Yanaco New Science Inc., Fushimi-ku, Kyoto, Japan) (for fluorine). Thermal analysis was performed on a SII DSC7020 with an electric cooling unit.

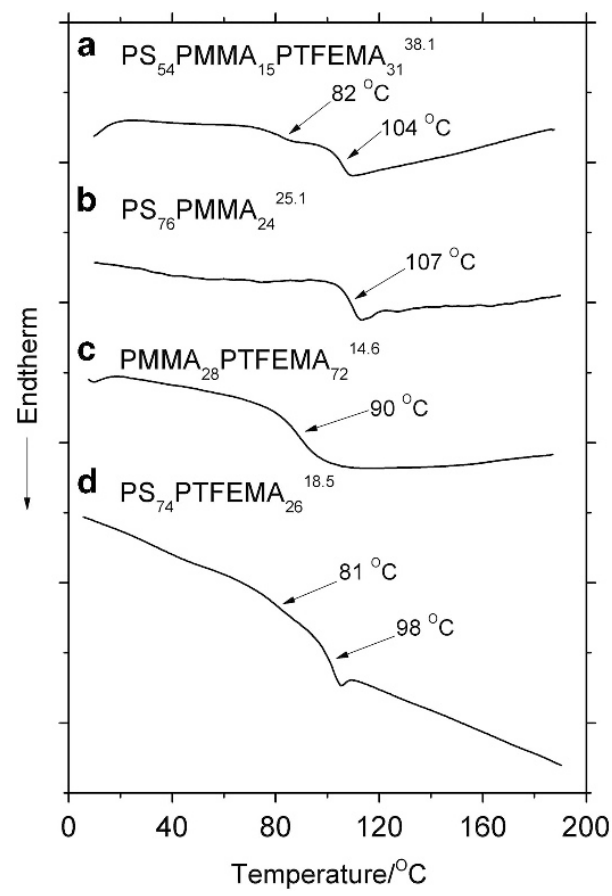

Figure 2 DSC traces of the second heating cycle for $\mathrm{PS}_{54} \mathrm{PMMA}_{15}$ PTFEMA $_{31}{ }^{38.1}$ (a), PS $_{76}$ PMMA $_{24}{ }^{25.1}$ (b), and PMMA $_{28}$ PTFEMA $_{72}{ }^{14.6}$ (c), and $\mathrm{PS}_{74} \mathrm{PTFEMA}_{26}{ }^{18.5}$ (d). 

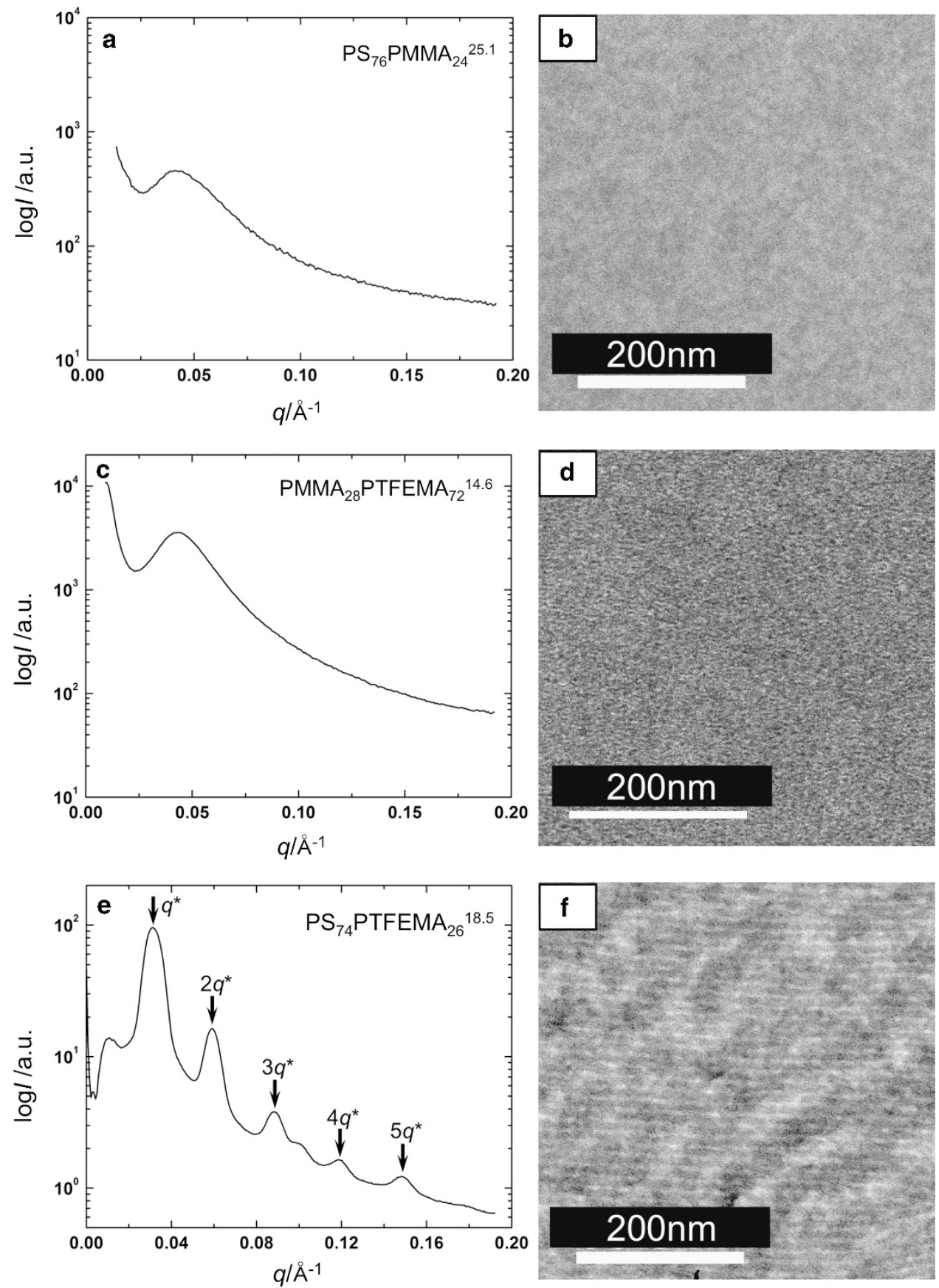

Figure 3 SAXS patterns and TEM images for the bulk samples of $\mathrm{PS}_{76} \mathrm{PMMA}_{24}{ }^{25.1}$ (a, b), $\mathrm{PMMA}_{28} \mathrm{PTFEMA}_{72}{ }^{14.6}$ (c, d), and PS ${ }_{74} \mathrm{PTFEMA}_{26}{ }^{18.5}$ $(e, f)$ at room temperature. For TEM observation, $\mathrm{PS}_{76} \mathrm{PMMA}_{24}{ }^{25.1}$ and $\mathrm{PS}_{74} \mathrm{PTFEMA}_{26}{ }^{18.5}$ were stained with $\mathrm{RuO}_{4}$.

\section{SAXS measurements}

Synchrotron radiation (SR)-SAXS measurement for $\mathrm{PS}_{54} \mathrm{PMM}_{15} \mathrm{PTFEMA}_{31}{ }^{38.1}$, as shown in Figure 4, was performed at the BL-10C beamline at the Photon Factory (PF), Tsukuba, Japan equipped with a 1D position sensitive proportional counter with 512 channels with a camera length of approximately $2 \mathrm{~m}$. The X-ray wavelength was $l=1.488 \AA$. Other samples of diblock copolymer were measured on a Bruker AXS NanoSTAR with a 2D CCD camera operating with $\mathrm{CuK} \alpha$ radiation. The X-ray wavelength was $l=1.54 \AA$. The sample-to-detector distance was approximately $1055 \mathrm{~mm}$. The scattering intensity was corrected by transmission and subtraction of background scattering and was plotted against the scattering vector $q=4 \pi \sin \theta / \lambda$, where $\theta$ was half of the scattering angle.

\section{SEM analysis}

The nanoporous structure shown in Figure 4c was imaged using a Hitachi S-4800 field-emission scanning electron microscope (SEM) operated with an acceleration voltage of $1.5 \mathrm{kV}$.

\section{Sample preparation for TEM and TEMT analysis}

Ultrathin $(30-80 \mathrm{~nm})$ samples for TEM and TEMT experiments were cut with a microtome. Ultrathin films were stained with $\mathrm{RuO}_{4}$. For TEMT analysis, 


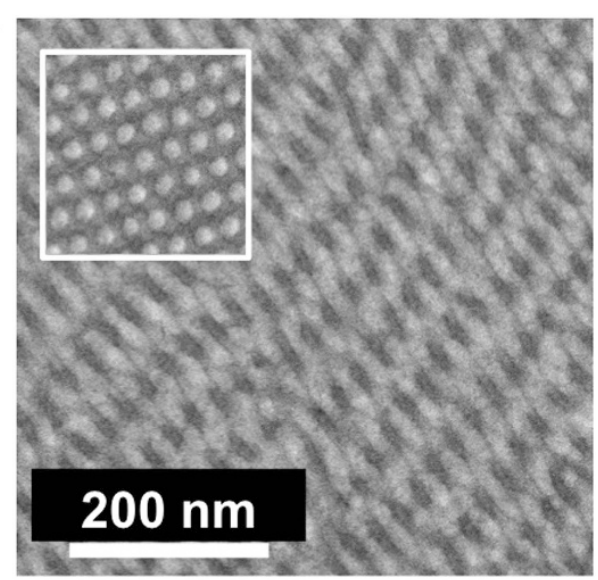

b
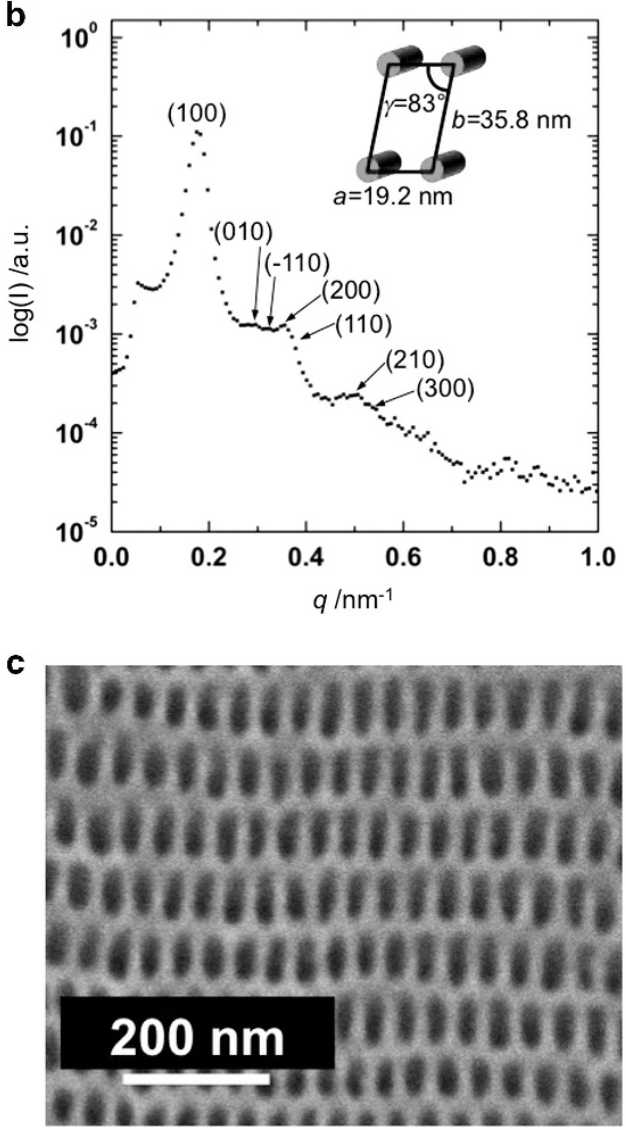

Figure 4 (a) TEM micrographs of $\mathrm{PS}_{54} \mathrm{PMMA}_{15} \mathrm{PTFEMA}_{31} 38.1$ bulk slices after treatment with $\mathrm{RuO}_{4}$, (b) A SR-SAXS profile acquired on a 1D detector of the sample, and (c) SEM image of PS ${ }_{54}$ PMMA $_{15}$ PTFEMA $_{31}{ }^{38.1}$ after DUV irradiation followed by methanol rinse. The inset image in (a) shows a cylindrical array of light-colored region close to the perpendicular beam incidence. Inset image of (b) is a schematic sketch of the oblique-packed cylindrical domains. A full color version of this figure is available at Polymer Journal online.

a vacuum staining system (Filgen Inc., Japan) was used. Au nanoparticles (diameter: $5 \mathrm{~nm}$ ) were placed on the supporting membrane of the TEM grid.

\section{TEM analysis}

Brightfield (BF)-TEM images were taken with a JEOL JEM1010BS operated at an acceleration voltage of $80 \mathrm{kV}$. The data were corrected with an imaging plate (IP).

\section{Dual-axis TEMT analysis}

The TEMT experiments were performed on a JEOL JEM-2200FS operated at $200 \mathrm{kV}$. The digital data of the transmitted images were collected with a slow-scan CCD camera (USC4000, Gatan Inc., USA). Two sets of tilt series were taken at the same area of the specimen. A projection at each tilt angle was acquired with a frame size of 2048 by 2048 pixels. To obtain achromatic projections, only the transmitted and elastically scattered electrons (electron energy loss: $0 \pm 60 \mathrm{eV}$ ) were selected by an energy filter $(\Omega$ filter, JEOL, Japan) installed in a JEM-2200FS. The first series was taken over the tilt angle from -62.7 to 60.7 with $1^{\circ}$ increment. After the first series was completed, the $\mathrm{Cu}$ mesh grid was manually rotated by approximately $90^{\circ}$, and the second tilt series was acquired over the tilt angles ranging from -58.4 to 52.8 with $1^{\circ}$ increment. The pixel size was 0.586 by $0.586 \mathrm{~nm}$. Prior to computing a 3D reconstruction, an alignment of the tilt series was necessary because there is an uncontrolled and magnification-dependent rotation of the projection by the electron optics within the microscope. This alignment also determines the tilt axis within the TEM micrographs and corrects any miscalibration in the goniometer stage. Each tilt series was aligned separately using $5 \mathrm{~nm}$ gold particles placed on the section as fiducial markers. An area common to each projection in the tilt series was utilized to perform the alignment by tracing the motions of several common fiducial markers (Au nanoparticles) in the area. The mean alignment error, averaged over all of the fiducial markers used in the alignment, was less than the pixel resolution, regardless of the tilt angle. The tilt series after the alignment were subsequently reconstructed by the filtered back-projection algorithm. Combining 3D reconstructions from two tilt series is described elsewhere. ${ }^{25}$ Visualizing software Amira (VSG, France) was used to obtain the $3 \mathrm{D}$ reconstructed image.

\section{RESULTS AND DISCUSSION}

The target triblock terpolymer was prepared by anionic polymerization of styrene, methyl methacrylate and 2,2,2-trifluoroethyl methacrylate in THF with sec-butyllithium as an initiator at $-78^{\circ} \mathrm{C}$ under an argon atmosphere (Figure 1). The primary structure of the resulting block copolymer was characterized by ${ }^{1} \mathrm{H}$ NMR and ${ }^{13} \mathrm{C}$ NMR spectroscopies, as shown in Supplementary Figure S1. After the polymerization of styrene, an aliquot was characterized by GPC for the molecular weight characterization. Supplementary Figure S2 shows the GPC diagrams of a triblock terpolymer and its polystyrene precursor. The resulting chromatograms show that the molecular weight of the triblock terpolymer was increased compared to the polystyrene precursor, as expected, and a narrow molecular weight distribution was obtained for each step. The molecular weight was $38.1 \mathrm{~kg} / \mathrm{mol}$, and the degrees of polymerization were 174 (PS), 57 (PMMA), and 85 (PTFEMA), as determined by a combination of the absolute molecular weight of PS and elemental analysis. Volume fractions were calculated from the degree of polymerization and the density of the each homopolymer. ${ }^{23}$

The phase behavior of $\mathrm{PS}_{54} \mathrm{PMMA}_{15} \mathrm{PTFEMA}_{31}{ }^{38.1}$, where the subscripts denote the volume fraction of the corresponding block and the superscript denotes the overall molecular weight $\left(M_{\mathrm{n}}\right)$ in $\mathrm{kg} / \mathrm{mol}$, was investigated using DSC. The DSC curve in the second heating cycle of the $\mathrm{PS}_{54} \mathrm{PMMA}_{15} \mathrm{PTFEMA}_{31}{ }^{38.1}$ showed two distinctive baseline shifts at 82 and $104{ }^{\circ} \mathrm{C}$, corresponding to the $T_{\mathrm{g}}$ of PTFEMA and PS, respectively, whereas no clear baseline shift corresponding to the $T_{\mathrm{g}}$ of PMMA of approximately $130^{\circ} \mathrm{C}^{26}$ was observed, as seen in Figure 2. For more detailed information, three diblock copolymers, $\mathrm{PS}_{76} \mathrm{PMMA}_{24}{ }^{25.1}$, $\mathrm{PMMA}_{28} \mathrm{PTFEMA}_{72}{ }^{14.6}$, and $\mathrm{PS}_{74} \mathrm{PTFEMA}_{26}{ }^{18.5}$ were synthesized. The molecular weight and composition of each block segment of these diblock copolymer samples were almost the same as those of $\mathrm{PS}_{54} \mathrm{PMMA}_{15} \mathrm{PTFEMA}_{31}{ }^{38.1}$. The DSC curves in the second heating cycles for $\mathrm{PS}_{76} \mathrm{PMMA}_{24}{ }^{25.1}$ and $\mathrm{PMMA}_{28} \mathrm{PTFEMA}_{72}{ }^{14.6}$ showed only one baseline shift (Figures $2 \mathrm{~b}$ and c), implying that the microphase separation in these samples did 


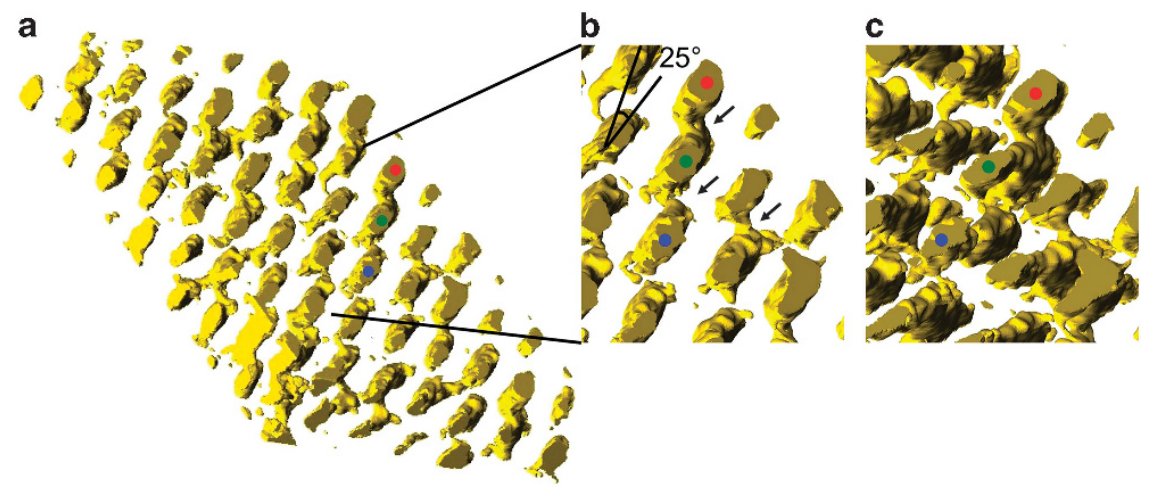

Figure 5 TEMT reconstructed image for bulk sample of $\mathrm{PS}_{54} \mathrm{PMMA}_{15} \mathrm{PTFEMA}_{31}{ }^{38.1}$ bulk slices after treatment with RuO 4 . Only light-colored domains are shown. An image-captured angle of this 3D image is almost parallel to the cylindrical structure (a); magnified image of (a, b); a different view of (b, c). The red, green and blue dots in each image represent the same cylinders among (a-c) for comparison.

not occur or was diffuse. The SAXS and TEM data of those diblock copolymers also indicated that $\mathrm{PS}_{76} \mathrm{PMMA}_{24}{ }^{25.1}$ and $\mathrm{PMMA}_{28}$ PTFEMA $_{72}{ }^{14.6}$ are miscible, whereas PS $_{74}$ PTFEMA $_{26}{ }^{18.5}$ showed wellordered microphase-separated structure (Figure 3). Based on these results, the short middle segment of PMMA does not form a distinct domain itself, as expected.

The bulk morphology formed in $\mathrm{PS}_{54} \mathrm{PMMA}_{15} \mathrm{PTFEMA}_{31}{ }^{38.1}$ was investigated. Bulk films of $\mathrm{PS}_{54} \mathrm{PMMA}_{15} \mathrm{PTFEMA}_{31}{ }^{38.1}$ were slowly cast from chloroform solutions (during approximately 4 days) and were subsequently annealed in saturated chloroform vapor at $100{ }^{\circ} \mathrm{C}$ for $4 \mathrm{~h}$. Figure $4 \mathrm{a}$ shows the bulk morphology of $\mathrm{PS}_{54} \mathrm{PMMA}_{15}$ PTFEMA $_{31}{ }^{38.1}$ stained with $\mathrm{RuO}_{4}$. Both dark- and light-colored domains showed ordered dot structures, both of which can be considered one of the facets of the ordered structure formed in this polymer. A corresponding SR-SAXS experiment was performed to further identify the observed morphology. The SR-SAXS profile acquired on a one-dimensional detector is shown in Figure $4 \mathrm{~b}$. The resulting plots produced peaks located at $q / q^{*}=1,1.69,1.86,2.00$, $2.07,2.46,2.77$, and 3.00. This sequence does not conform to a cubic, tetragonal, or hexagonal Bravais lattice. The analysis of the pattern was performed on the basis of the following equation: ${ }^{27}$

$$
\frac{q^{2}}{(2 \pi)^{2}}=\frac{h^{2}}{(a \sin \gamma)^{2}}-\frac{2 h k \cos \gamma}{a b \sin ^{2} \gamma}+\frac{k^{2}}{(b \sin \gamma)^{2}}
$$

where $h$ and $k$ are the Miller indices of the scattering planes, $a$ and $b$ are the unit cell basis vectors, and $\gamma$ is the angle between $a$ and $b$ $\left(0^{\circ}<\gamma<180^{\circ}\right)$. Using the above equation, the eight peaks were consistently indexed as (100), (010), (-110), (200), (110), (210) and (310), in agreement with a $2 \mathrm{D}$ oblique lattice with lattice parameters $a=19.2 \mathrm{~nm}, b=35.8 \mathrm{~nm}$ and $\gamma=83^{\circ}$. Here, $h$ and $k$ are the associated Miller indices, as illustrated in the inset of Figure $4 \mathrm{~b}$. From the SR-SAXS result, peaks based on only the periodic structure in the $h k$ plane were observed. Figure $4 \mathrm{c}$ shows the SEM image of the microtomed film on a silicon wafer after DUV irradiation followed by methanol rinse. The PTFEMA domain was selectively degraded by $0.8 \mathrm{~J} \mathrm{~cm}^{-2}$ of DUV to form a porous film.

In addition to the results in Figure 4, TEMT experiments were performed to investigate the three-dimensional (3D) structure of the $\mathrm{PS}_{54} \mathrm{PMMA}_{15} \mathrm{PTFEMA}_{31}{ }^{38.1}$. To obtain as quantitative a $3 \mathrm{D}$ image as possible, especially for the thickness direction of the thin film, dual-axis TEMT was performed. ${ }^{25}$ Figure 5 a displays a reconstructed $3 \mathrm{D}$ image of $\mathrm{PS}_{54} \mathrm{PMMA}_{15} \mathrm{PTFEMA}_{31}{ }^{38.1}$. For the sake of clarity, the light gray region in Figure $4 \mathrm{a}$ is shown in yellow color in the images.
The domain lattice parameters obtained from this $3 \mathrm{D}$ image were $a \sim 17 \mathrm{~nm}, b \sim 35 \mathrm{~nm}$ and $\gamma \sim 86^{\circ}$, which were in excellent agreement with those obtained from the SAXS experiments: $a=16.2 \mathrm{~nm}$, $b=35.8 \mathrm{~nm}$ and $\gamma=83^{\circ}$. As seen in the magnified image in Figures $5 \mathrm{~b}$ and $\mathrm{c}$, elliptical cylinders whose major radius axis tilted approximately $25^{\circ}$ with respect to the $y$-axis were observed and were partially continuous (indicated by arrows in Figure $5 b$ ). The volume fraction of the yellow-colored domain was $23 \pm 2 \%$, which was lower than the known volume fraction of PTFEMA (31\%). Thus, the light gray region in the TEM image, i.e., the cylindrical structure with oblique lattice, can be assigned to mostly PTFEMA with a small amount of PMMA, whereas the dark gray region, i.e., the matrix, can be assigned to PS and the PMMA segment. This discrepancy can be considered small because of the relatively small observation volume of the 3D image compared with that of the unit cell (especially for the $\mathrm{z}$ direction). The threshold used to determine the interface between the cylinders and matrix may also explain the difference.

$\mathrm{PS}_{54} \mathrm{PMMA}_{15} \mathrm{PTFEMA}_{31}{ }^{38.1}$ did not form a lamellar structure, whereas the corresponding diblock copolymer PS- $b$-PTFEMA with almost the same volume of 31 vol\% PTFEMA formed a lamellar structure, as reported in our previous study. ${ }^{23}$ In the diblock copolymer PS-b-PTFEMA with 31 vol\% PTFEMA, the ordered lamellar structure largely reflects the factor to reduce the interfacial area due to the strong repulsive force between PS and PTFEMA. In contrast, the partially continuous cylindrical structure with oblique lattice formed in $\mathrm{PS}_{54} \mathrm{PMMA}_{15} \mathrm{PTFEMA}_{31}{ }^{38.1}$ is more reflective of other factors, including conformational entropy, compressibility, molecular rigidity, and details of the molecular interaction due to the manipulated interface (weakened interfacial energy by a short PMMA middle segment). A columnar structure with an oblique lattice is reported in dendritic polymers or molecules with liquid crystal or crystal properties, ${ }^{27-29}$ in which the authors argued these morphologies are dominated by their crystallinity or liquid crystallinity. In our case, PTFEMA is neither liquid crystal nor crystal, but it is reasonable that molecular rigidity and/or details of the intermolecular interaction due to the polar trifluoroethyl group ${ }^{23}$ are significant factors in the formation of partially continuous cylindrical structures with an oblique lattice.

We also infer that the short PMMA middle segment has an important role as a trigger in interfacial mixing and lowering the degree of segregation of the PS and PTFEMA segments. The weakened segregation most likely induced the formation of two-phase type microphase-separated nanostructure that is PS-rich and PTFEMA-rich microdomains with the unconventional partially cylindrical 
morphology. This morphology was not achieved by the combination of PS and PTFEMA alone. The PMMA segment in the $\mathrm{PS}_{54} \mathrm{PMMA}_{15} \mathrm{PTFEMA}_{31}{ }^{38.1}$ did not form its own domain because of its low molecular weight. Instead, the PMMA segment dissolved in both PS and PTFEMA microdomains and broadened the interface between the two domains.

In summary, we successfully demonstrated the creation of unconventional block copolymer morphology by manipulating the interface with a short middle segment in a triblock copolymer. The target triblock terpolymer, $\mathrm{PS}_{54} \mathrm{PMMA}_{15} \mathrm{PTFEMA}_{31}{ }^{38.1}$, was synthesized via sequential living anionic polymerization and was found to form partially continuous oblate cylinders with an oblique lattice that were not obtained in the previously reported diblock copolymer PS- $b$-PTFEMA or other linear block copolymers. The role of the PMMA resembles the tapered block segments reported by Hashimoto and coworkers, ${ }^{17}$ but our chemical design simplified the synthetic procedure and expanded the variety of block copolymer with a modified interface. The introduction of a short middle block segment in $\mathrm{ABC}$ type triblock terpolymers provides a unique opportunity to modify the interfacial morphologies between the A and $\mathrm{C}$ domains. Such interfacial manipulation by the presence of the middle short block segment is an essential strategy to create a new class of two- or multi-phase type microphase-separated nanostructures.

\section{CONFLICT OF INTEREST}

The authors declare no conflict of interest.

\section{ACKNOWLEDGEMENTS}

Portions of this research were conducted at the Center for Advanced Materials Analysis (CAMA) at Tokyo Institute of Technology and the Photon Factory (PF) Tsukuba, Japan. The authors thank Prof M Tokita and Ms M Koga at Tokyo Institute of Technology for the SR-SAXS measurement and assignment, and Dr H Yoshida, Dr K Aida at Hitachi for the SEM imaging. The authors also thank Prof T Hashimoto and Prof M Takenaka at Kyoto University and Dr H Sugimori at KRI for helpful discussions. RM acknowledges support from the Japan Society for the Promotion of Science (JSPS) as well as Cornell University. HJ is grateful to the Ministry of Education, Science, Sports and Culture through Grants-in-Aid No. 24310092. CKO is grateful to the NSF (DMR-1506542) for partial support of this work. TH is grateful to the Japan Science and Technology Agency (JST), Precursory Research for Embryonic Science and Technology (PRESTO) on the Molecular Technology and Creation of New Functions.

1 Park, M., Harrison, C., Chaikin, P. M., Register, R. A. \& Adamson, D. H. Block Copolymer Lithography: Periodic Arrays of $\sim 10^{11}$ Holes in 1 Square Centimeter. Science 276, 1401-1404 (1997).

2 Hirai, T., Leolukman, M., Liu, C. C., Han, E., Kim, Y. J., Ishida, Y., Hayakawa, T., Kakimoto, M.-a., Nealey, P. F. \& Gopalan, P. One-step direct patterning template utilizing self-assembly of POSS containing block copolymers. Adv. Mater. 21, 4334-4338 (2009).

3 Morita, H. Lithography process simulation studies using coarse-grained polymer models. Polym. J. 48, 45-50 (2016).

4 Ueno, K., Fukai, T. \& Watanabe, M. Thermosensitive soft glassy colloidal arrays of block copolymer-grafted silica nanoparticles in an ionic liquid. Polym. J. 48, 289-294 (2016).
5 Breiner, U., Krappe, U., Thomas, E. L. \& Stadler, R. Structural Characterization of the 'Knitting Pattern' in Polystyrene-block-poly(ethylene-co-butylene)-block-poly(methyl methacrylate) Triblock Copolymers. Macromolecules 31, 135-141 (1998).

6 Krappe, U., Stadler, R. \& Voigt-Martin, I. Chiral Assembly in Amorphous ABC Triblock Copolymers. Formation of a Helical Morphology in Polystyrene-block-polybutadieneblock-poly(methyl methacrylate) Block Copolymers. Macromolecules 28, 4558-4561 (1995)

7 Hong, S., Higuchi, T., Sugimori, H., Kaneko, T., Abetz, V., Takahara, A. \& Jinnai, H.. Highly oriented and ordered double-helical morphoology in $A B C$ triblock terpolymer films up to micrometer thickness by solvent evaporation. Polym. J. 44 , 567-572 (2012)

8 Abetz, V. \& Simon, P. F. W. Phase Behaviour and Morphologies of Block Copolymers. Adv. Polym. Sci. 189, 125-212 (2005).

9 Abetz, V. \& Goldacker, T. Formation of superlattices via blending of block copolymers. Macromol. Rapid Commun. 21, 16-34 (2000).

10 Matsushita, Y., Hayashida, K. \& Takano, A. Jewelry Box of Morphologies with Mesoscopic Length Scales - ABC Star-shaped Terpolymers. Macromol. Rapid Commun. 31, 1579-1587 (2010).

11 Ungar, G., Liu, Y. S., Zeng, X. B., Percec, V. \& Cho, W. D. Giant Supramolecular Liquid Crystal Lattice. Science 299, 1208-1211 (2003).

12 Cho, B. K., Jain, A., Gruner, S. M. \& Wiesner, U. Mesophase Structure-Mechanical and Ionic Transport Correlations in Extended Amphiphilic Dendrons. Science 305, 1598-1601 (2004).

13 Maeda, R., Hayakawa, T., Tokita, M., Kikuchi, R., Kouki, J., Kakimoto, M.-a. \& Urushibata, H. Double liquid crystalline side-chain type block copolymers for hierarchically ordered nanostructures: Synthesis and morphologies in the bulk and thin film. React. Funct. Polym. 69, 519-529 (2009).

$14 \mathrm{Ma}$, X., Usui, R., Kitazawa, Y., Kokubo, H. \& Watanabe, M. Temperature and light-induced self-assembly changes of a tetra-arm diblock copolymer in an ionic liquid. Polym. J. 47, 739-746 (2015).

15 Tsukahara, Y., Nakamura, N., Hashimoto, T., Kawai, H., Nagaya, T., Sugimura, Y. \& Tsuge, S. Structure and Properties of Tapered Block Polymers of Styrene and Isoprene. Polym. J. 12, 455-466 (1980).

16 Hashimoto, T., Tsukahara, Y. \& Kawai, H. Structure and Properties of Tapered Block Polymers of Styrene and Isoprene II. Dynamic Mechanical Responses and Their Structural Interpretations. Polym. J. 15, 699-711 (1983).

17 Hashimoto, T., Tsukahara, Y., Tachi, K. \& Kawai, H. Structure and properties of tapered block polymers. 4. 'Domain-boundary mixing' and 'mixing-in-domain' effects on microdomain morphology and linear dynamic mechanical response. Macromolecules 16, 648-657 (1983).

18 Hasegawa, H., Hashimoto, T. \& Hyde, S. T. Microdomain structures with hyperbolic interfaces in block and graft copolymer systems. Polymer 37, 3825-3833 (1996).

19 Hodrokoukes, P., Floudas, G., Pispas, S. \& Hadjichristidis, N. Microphase Separation in Normal and Inverse Tapered Block Copolymers of Polystyrene and Polyisoprene. 1. Phase State. Macromolecules 34, 650-657 (2001).

20 Singh, N., Tureau, M. S. \& Epps, T. H. Manipulating ordering transitions in interfacially modified block copolymers. Soft Matter 5, 4757-4762 (2009).

21 Roy, R., Park, J. K., Young, W. S., Mastroianni, S. E., Tureau, M. S. \& Epps, T. H. Double-Gyroid Network Morphology in Tapered Diblock Copolymers. Macromolecules 44, 3910-3915 (2011).

22 Kuan, W. F., Roy, R., Rong, L. X., Hsiao, B. S. \& Epps, T. H. Design and Synthesis of Network-Forming Triblock Copolymers Using Tapered Block Interfaces. ACS Macro Lett. 1, 519-523 (2012).

23 Maeda, R., Hayakawa, T. \& Ober, C. K. Dual Mode Patterning of Fluorine-Containing Block Copolymers through Combined Top-down and Bottom-up Lithography. Chem. Mater. 24, 1454-1461 (2012).

24 Jinnai, H., Spontak, R. J. \& Nishi, T. Transmission Electron Microtomography and Polymer Nanostructures. Macromolecules 43, 1675-1688 (2010).

25 Sugimori, H., Nishi, T. \& Jinnai, H. Dual-Axis Electron Tomography for ThreeDimensional Observations of Polymeric Nanostructures. Macromolecules 38, 10226-10233 (2005).

26 Andrews, R. J. \& Grulke, E. A. in Polymer Handbook, 4th edn (eds Brandrup, J., Immergut, E. H. \& Grulke, E. A.) Ch. VI, 193-277 (John Wiley \& Sons, Hoboken, NJ, USA, 1999).

27 Lee, E., Lee, B. I. Kim, S. H. Lee, J. K., Zin, W. C. \& Cho, B. K. Complex Thermal and Bulk Assembling Properties of Dendritic-Linear-Dendritic Triblock Copolymers Depending on the Length of the Middle Block. Macromolecules 42, 4134-4140 (2009).

28 Hanski, S., Junnila, S., Soininen, A. J., Ruokolainen, J. \& Ikkala, O. Oblique Self-Assemblies and Order - Order Transitions in Polypeptide Complexes with PEGylated Triple-Tail Lipids. Biomacromolecules 11, 3440-3447 (2010).

29 Sengupta, S., Uemura, S., Patwardhan, S., Huber, V., Grozema, F. C., Siebbeles, L. D. A, Baumeister, U. \& Würthner, F.. Columnar Mesophases Based on Zinc Chlorophyll Derivatives Functionalized with Peripheral Dendron Wedges. Chem. Eur. J 17, 5300-5310 (2011).

Supplementary Information accompanies the paper on Polymer Journal website (http://www.nature.com/pj) 